\title{
IMPLICATIONS OF ANTENNA SYSTEM CALIBRATION ON SPACECRAFT DESIGN AND RADIO DATA ANALYSIS
}

\author{
H. O. Rucker*, M. Sampl*, M. Panchenko*, T. H. Oswald*, \\ D. Plettemeier ${ }^{\dagger}$, M. Maksimovic ${ }^{\ddagger}$, and W. Macher*
}

\begin{abstract}
Currents on the conducting surfaces of the spacecraft hull, induced by electric fields of radio waves, strongly influence the reception properties of spacecraft antenna systems. This influence is visualized by the so-called "effective antenna length" $\left(\mathbf{h}_{e f f}\right)$, representing the electric antenna, which differs from the physical antenna rod. Knowledge on these effective antenna vectors can be yielded by several different methods: (1) Experimental rheometry, (2) Numerical computer simulations, (3) In-flight calibration, and (4) Experimental anechoic chamber measurements.

The paper addresses these methods and shows in the case of preliminary design studies of Solar Orbiter spacecraft the possibilities of numerical computer simulations, in particular the change of $\mathbf{h}_{e f f}$ by design variations. The combined use of the above mentioned methods enables the determination of $\mathbf{h}_{\text {eff }}$ over a wide frequency range, the correct information on wave polarisation, and in specific cases helps to improve the performance of direction finding. So the calibration results may also be used to re-evaluate structure and position of antennas (and even positions of instruments) on board a spacecraft.
\end{abstract}

\section{The Concept of Effective Length Vector of an Antenna}

The reception properties of antennas aboard a spacecraft are strongly influenced by the complex structure of the metallic configuration of the spacecraft body, leading to a distortion of the "electric antennas" with regard to the physical antenna rods. Assuming an electromagnetic wave with electric field $\mathbf{E}$ incident on an antenna, the received voltage is a function of both the $\mathbf{E}$-field and the effective length vector $\mathbf{h}_{\text {eff }}$ :

\footnotetext{
* Space Research Institute, Austrian Academy of Sciences, Schmiedlstrasse 6, A-8042 Graz, Austria

$\dagger$ Technical University Dresden, Chair in RF and Photonics, Georg-Schumann-Strasse 9, 01187 Dresden, Germany

$\ddagger$ LESIA, Observatoire de Paris, UMR CNRS 8109, 92195 Meudon, France
} 


$$
\begin{gathered}
V=\mathbf{E} \cdot \mathbf{h}_{e f f} \\
\mathbf{h}_{e f f}=\frac{1}{I_{a}} \int \mathbf{J}\left(\mathbf{r}^{\prime}\right) e^{j \mathbf{k} \cdot \mathbf{r}} \mathrm{d} V^{\prime}
\end{gathered}
$$

The effective antenna length vector $\mathbf{h}_{\text {eff }}$ is the integral of the surface current density amplitude $\mathbf{J}$ around the volume $V^{\prime}, I_{a}$ denotes the port current driving the antenna [Ortega-Molina and Daigne, 1984; Balanis, 2005; Sampl et al., 2011a; this issue 2011b]. Usually the vector $\mathbf{h}_{\text {eff }}$ is a complex quantity, but only in the quasi-static case $(\lambda>>d$, wavelength $\lambda$, geometrical scale length $d$, e.g. antenna length) $\mathbf{h}_{\text {eff }}$ can be treated as a real vector and is independent from the direction of incident waves on the antenna rod.

\section{Antenna System Calibration Methods}

Based on experimental methods (rheometry, anechoic chamber measurements, in-flight calibration) as well as on theoretical / numerical computer simulations the reliable determination of the effective length vector $\mathbf{h}_{e f f}$ is constrained within certain frequency ranges. Although in principle direction finding is possible above the quasistatic frequency range, reliable results can only be expected far below the first resonance, because the $\mathbf{h}_{\text {eff }}$ is very sensitive even to tiny frequency uncertainties near the resonance. Therefore usually direction finding has been performed only for wavelengths which are much greater than the effective length $\left(\left|\mathbf{h}_{e f f}\right| \ll c / \omega\right)$. The experimental rheometry method, in detail described in Rucker et al. [1996], Macher et al. [2007], and Oswald et al. [2009], uses a spacecraft modelled to scale, and immersed in an electrolytic (water) tank, measures the antennas voltage response to an externally applied homogeneous electric field, stimulating the incident radio waves. Meaningful results of $\mathbf{h}_{\text {eff }}$ can be obtained at radio frequencies between a few tens of $\mathrm{kHz}$ up to approx. 1.5 MHz.

Contrary to the experimental rheometry method, where the plane wave impinging on the spacecraft model is simulated by an externally applied quasi-static (few $\mathrm{kHz}$ ) electric field, anechoic chamber measurements (ACM) use microwaves of hundreds of $\mathrm{MHz}$ up to several $\mathrm{GHz}$ radiated towards a frequency scaled spacecraft model. In the anechoic chamber the spacecraft model is linearly scaled with the signal wavelength and placed on a three axes computer controlled positioner unit. The reception properties of the spacecraft antenna system are depending on frequency, polarization, and angle of incidence. The frequency scaled signal is transmitted by a fully polarimetric antenna system located in the far field of the spacecraft model. In the course of ACM radiation coupling and scattering effects occur at the spacecraft, and this can be characterized and determined in dependence of radiated frequency and wave polarization. Interferences are minimized by using an electromagnetic shielded chamber and specifically shaped absorbers at the surrounding walls. Under the assumption of, e.g., a $30 \mathrm{MHz}$ radio burst signal received by a real spacecraft antenna system, the ACM radiating on a 1:30 scale model has to perform the simulation using a $900 \mathrm{MHz}$ radio wave [Baganz et al., 2008; Bucci et al., 2009; Landmann et al., 2006]. Corresponding anechoic chamber measurements are at present prepared together with our partner at University of Dresden within the project calibrating 
the NASA/JUNO/WAVES antenna system in order to obtain experimentally supported results on the antenna reception lobes also at higher frequencies (i.e. $30 \mathrm{MHz} \leq f \leq 40$ $\mathrm{MHz})$.

As a third "experimental" method the "in-flight calibration" can be regarded as the most reliable method to determine the reception properties of a spacecraft antenna system since the "real" spacecraft configuration is involved. In-flight calibration of the Cassini-RPWS antenna system has been performed prior to and after the Jupiter flyby of Dec 2000. At some distance the radio source can be regarded as point source (i.e. the position of Jupiter), and by spacecraft rotation around a defined axis the corresponding antenna responses (analytic antenna signals and their real/imaginary auto-/crosscorrelation products) can be analysed in order to retrieve the corresponding effective antenna length [Vogl et al., 2004; Cecconi and Zarka, 2005].

A further in-flight calibration has recently been treated on the STEREO/WAVES antennas by using the terrestrial radio emission (AKR), when STEREO-B made a corresponding rotation manoeuvre in January 2007 [Panchenko et al., 2010]. Again, with the distant location of STEREO-B relative to the Earth, the AKR sources can be assumed to coincide with the position of the Earth's center as known parameter. The unknown parameters however are the 3 effective length vectors of the $\mathrm{x}-, \mathrm{y}$-, and $\mathrm{z}$-antennas, the colatitude and azimuth parameters of the 3 antennas and (for checking-reasons) the 4 Stokes parameters, in total 13 unknowns. The applied genetic code [Panchenko et al., 2010] yielded coinciding results both in the case of calculation with the 13 unknowns as well as in the case using the known 4 Stokes Parameters for AKR (then only with 9 unknowns).

The most versatile method, considering effort and financial input, is the numerical computer simulation, as outlined in more detail in the subsequent chapter. The numerical calibration fully displays the possibility to check the influence of the spacecraft configuration on the reception properties of the antenna system, and enables to "play" with different designs and look for the corresponding $\mathbf{h}_{\text {eff }}$. Table 1 summarizes past, present and future antenna system calibrations.

It is important to estimate the accuracy of the different calibration methods. The rheometry method essentially comprises two sources of error: the exact modeling of the spacecraft, specifically around the area of antenna footpoints, and secondly, the exact reading of the angles in the course of performance of rheometry measurements with the spacecraft model immersed in a water tank. Both errors could add up to 1 to 2 degrees of uncertainty in the directions of the effective antenna vectors. Numerical computer simulations also strongly depend on the accuracy of modeling, which may result in errors up to 1 degree [Macher and Oswald, 2011]. The most accurate method of cause is the inflight calibration which "uses" the real spacecraft. Even here, however, errors have to be considered, mainly arising from low signal/noise levels and from the applied statistics in the data analysis, leading to method errors of $<1$ degree. ACM measurements uncertainties may be comparable to those of numerical simulations. 


\section{Numerical Computer Simulation of Antenna System Calibra- tion}

At the beginning of each numerical computer simulation is the mathematical spacecraft design by wiregrid or in combination with patchgrid modelling. Figure 1 displays the Solar Orbiter wiregrid modelling as used for the subsequent analysis of the antenna system reception properties. Note that a CAD model as well as a polygon mesh model for Solar Orbiter was used in the paper by Sampl et al. [this issue 2011b, Figures 1 and 2].

In order to avoid duplicating informations on the mathematical background and on the mechanical geometry of the Solar Orbiter reference is given to Sampl et al. [this issue 2011b, Eqn. (1), and Table (1)].

The present paper concentrates on the numerical simulation to optimize eventual direction finding possibilities as well as to study situations when the Solar Orbiter spacecraft is in cruise and operational phase.

Since Solar Orbiter will approach the Sun at rather close distance $(\approx 0.28 \mathrm{AU})$, the mechanical antenna rods are assumed to suffer thermal bending away from the Sun due to solar heating. Most likely the tip of each antenna rod is thermally bent by about $0.5 \mathrm{~m}$ off the nominal position in the anti-solar direction (Fig. 1). As outlined by Table 2 the effective antenna lengths of all three antennas ANT1, ANT2, ANT3 are bent towards the Sun (i.e. the positive $\mathrm{x}$-axis with $\theta=0$ is pointing towards the Sun), even in the case that their physical direction (antenna tip) is out of the coplanar plane by $0.5 \mathrm{~m}$ in the anti-solar direction. It is interesting to note that the directions of $\mathbf{h}_{\text {eff }}$ are tilted (for open feeds and nominal antenna footpoint position) by about 8-10 degrees, towards the Sun, out of the plane vertical to the $\mathrm{x}$-axis, which contains all three mechanical antenna rods.

The optimum antenna configuration for direction finding is of course an antenna system of two perpendicular antennas on a spinning spacecraft or three perpendicular antennas on a three-axis stabilized spacecraft. The original Solar Orbiter antenna system design provides all three mechanical antennas coplanar, and only the effective antenna lengths are slightly tilted to each other, with maximum tilt at the nominal antenna footpoint position.

In trying to increase the tilt of all three $\mathbf{h}_{\text {eff }}$ out of the plane containing the three mechanical antenna rods, for the purpose to optimize the conditions for direction finding, one possibility may be the shift of antenna footpoints on the spacecraft hull. The nominal antenna footpoint position (at present $235 \mathrm{~mm}$ distant from the heat shield in $-x$-direction) is already the closest possible position to the heat shield, so any variation of footpoint position can only be done in the anti-solar direction, performed in steps of $100 \mathrm{~mm}$.

As can be seen in Table 2 the angle $\theta$ widens (the tilt out of the plane containing all three antennas minimizes). This can be explained by the decrease of surface currents pushing the $\mathbf{h}_{\text {eff }}$ towards the positive x-axis.

As a resumé the closest possible position of the antenna footpoints to the heat shield is the optimum configuration for direction finding, if all three antenna rods have to keep coplanarity. Recent studies by Krupar et al. [this issue, 2011] have been performed on 


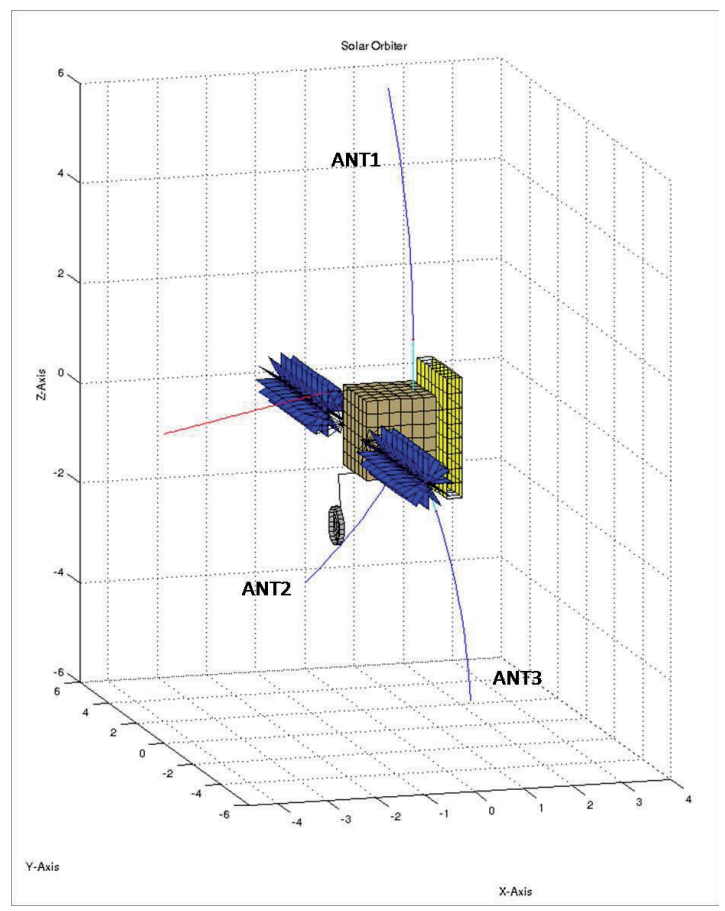

Figure 1: Wiregrid model of Solar Orbiter as used for the analysis of the antenna system calibration. The solar panels are drawn in superposition of various orientations (optimizing input of solar radiation) in the course of cruise/operational phase.

the goniopolarimetric inversion (direction finding and polarization determination from observed data) applied to solar type III radio bursts recorded by the RPW instrument of Solar Orbiter. Here the effective antenna tilt (out of the plane of coplanarity) and the variations of $\Delta \mathbf{k}$ (wave vector) and $\left|\mathbf{h}_{\text {eff }}\right|$ (antenna length error) have been brought to relation to each other. As a result Krupar et al. [this issue, 2011; 2010] concluded that an effective antenna tilt angle of at least 5-10 degrees is needed in order to obtain a direction finding accuracy better than 10 degrees including reasonable experimental uncertainties.

Further analysis with design variations like varying solar panel orientations (as indicated in Figure 1) or the rotation of the high-gain antenna in the course of orbital motion around the Sun only yields negligible effects of the $\theta$ - and $\phi$-variations of $\mathbf{h}_{\text {eff }}$ (Numbers are changing only well below the uncertainty level of about 1 degree.).

One aspect however is still open and in this paper not addressed at all: The (eventual) influence of the surrounding plasma on the reception properties of the antenna system. As investigated by Oswald [2010] there are effects by the surrounding plasma, initiating a plasma sheath around the spacecraft. Since the antennas are electromagnetically coupled to each other, this effect mirrors into the mutual resistance and capacitance describing 
Table 1: Spacecraft antenna system calibrations (encircled numbers indicate corresponding references, $A C M=$ Anechoic Chamber Measurements).

\begin{tabular}{|c|c|c|c|c|}
\hline Spacecraft & Rheometry & $\begin{array}{c}\text { Num. } \\
\text { calibration }\end{array}$ & In-flight cal. & $\mathrm{ACM}$ \\
\hline$\underset{\text { Voyager/PRA }}{t}$ & & (27) & (6) (7) 8 (13) (30) & (19) \\
\hline Cassini/RPWS & (20) (21) & (4) (21) & (5) (28) (29) & \\
\hline$\underset{\text { Mars }}{\text { Express/MARSIS }}$ & & (10) (11) & & \\
\hline & (24) (25) (26) & (24) (26) & & \\
\hline Interball/Polrad & & & (17) & \\
\hline STEREO/WAVES & (1) (9) (12) (16) & $\begin{array}{l}1123 \\
\text { (9) (14) (16) }\end{array}$ & (18) & (1) (31) \\
\hline $\begin{array}{c}\text { Solar } \\
\text { Orbiter/RPW }\end{array}$ & & (15) 23 & & $\begin{array}{c}\text { in } \\
\text { preparation }\end{array}$ \\
\hline & \multicolumn{4}{|c|}{$\leftarrow$ in preparation $\rightarrow$} \\
\hline & & $\leftarrow$ in prepar & tion $\rightarrow$ & \\
\hline & & & & \\
\hline
\end{tabular}


Table 2: Effective antenna lengths of Solar Orbiter antennas ANT1, ANT2, ANT3 (antenna rod length $5 \mathrm{~m}$, nominal $\theta=90, \phi=0,-125,+125$, resp.) with the assumption of thermal bending $(500 \mathrm{~mm})$ and variation of antennas footpoint positions. Numerical simulations used CONCEPT II and was performed for open feeds (no connection to the receiver) as well as assuming a base capacitance (e.g. capacitance of cables, receiver input) of $70 \mathrm{pF}$. Top: No shift from nominal antenna footpoint position, Middle/Bottom: $100 \mathrm{~mm} / 200 \mathrm{~mm}$ shift in the anti-solar direction.

\begin{tabular}{|l||l|l|l||l|l|l|}
\hline \multicolumn{1}{|c||}{} & \multicolumn{3}{l||}{ Open feeds } & \multicolumn{3}{l|}{ Base cap. 70 pF } \\
\hline & Length/m & $\theta /{ }^{\circ}$ & $\phi /{ }^{\circ}$ & Length/m & $\theta /^{\circ}$ & $\phi /^{\circ}$ \\
\hline ANT1 & 4.25 & 81.9 & 0.1 & 1.75 & 83.2 & 0.1 \\
\hline ANT2 & 4.20 & 81.2 & -128.5 & 1.71 & 82.4 & -127.7 \\
\hline ANT3 & 4.20 & 80.2 & 128.3 & 1.71 & 82.4 & 127.8 \\
\hline
\end{tabular}

\begin{tabular}{|l||l|l|l||l|l|l|}
\hline \multicolumn{1}{|c||}{} & \multicolumn{3}{l||}{ Open feeds } & \multicolumn{3}{l|}{ Base cap. 70 pF } \\
\hline & Length/m & $\theta /^{\circ}$ & $\phi /^{\circ}$ & Length/m & $\theta /^{\circ}$ & $\phi /^{\circ}$ \\
\hline ANT1 & 4.23 & 83.1 & 0.1 & 1.75 & 84.2 & 0.1 \\
\hline ANT2 & 4.18 & 82.4 & -128.4 & 1.71 & 83.4 & -127.9 \\
\hline ANT3 & 4.19 & 82.2 & 128.3 & 1.71 & 83.5 & 127.8 \\
\hline
\end{tabular}

\begin{tabular}{|l||l|l|l||l|l|l|}
\hline \multicolumn{1}{|c||}{} & \multicolumn{3}{c||}{ Open feeds } & \multicolumn{3}{|c|}{ Base cap. 70 pF } \\
\hline & Length/m & $\theta /^{\circ}$ & $\phi /^{\circ}$ & Length/m & $\theta /^{\circ}$ & $\phi /^{\circ}$ \\
\hline ANT1 & 4.23 & 84.3 & 0.1 & 1.75 & 85.2 & 0.1 \\
\hline ANT2 & 4.16 & 83.6 & -128.5 & 1.70 & 84.5 & -127.9 \\
\hline ANT3 & 4.17 & 83.6 & 128.4 & 1.70 & 84.5 & 127.8 \\
\hline
\end{tabular}

the interaction between the antennas. If a surrounding plasma is considered, also the plasma sheath resistance and capacitance have to be taken into account. First analysis of this effect - in the case of STEREO/WAVES - shows small, but visible changes, in particular a decrease in the length of $\mathbf{h}_{\text {eff }}$ [Oswald, 2010]. Considering Solar Orbiter in a high density solar wind environment close to the Sun, or JUNO in a high density Jovian magnetospheric magnetoplasma, may certainly enhance the importance of corresponding studies.

\section{Conclusions}

As experienced in the past by performing a number of calibration analyses of spacecraft antenna systems, the configuration of the antennas is either conclusively defined or the design is matter of only marginal variations. In both cases the described and applied methods of calibration yield valuable results which are of importance for a reliable radio data analysis.

Acknowledgements. The authors would like to thank M.Y. Boudjada for valuable comments, V. Gubchenko and another referee for evaluating the paper, and M. Scherf for 
the layout. While preparing this $7^{\text {th }}$ proceedings volume "Planetary Radio Emissions VII" sad news reached us on the passing of Yolande Leblanc (Observatoire de Paris-Meudon). On behalf of the Department of Extraterrestrial Physics HOR would like to express his deep gratitude to her, having continuously received great scientific support.

\section{References}

Baganz, N., D. Plettemeier, and K.-H. Gonschorek, Pattern Deformation of a Marine Communications Antenna on board a Frigate during a Helicopter Approach Application of the Geometrical Matrix Recycling Method (GMRM), Proceedings on International Symposium on Electromagnetic Compatibility, 187-192, 2008.

Balanis, C. A., Antenna Theory: Analysis and Design, 3rd Ed., John Wiley \& Sons, Inc, 2005.

Bale, S. D., R. Ullrich, K. Goetz, N. Alster, B. Cecconi, M. Dekkali, N. R. Lingner, W. Macher, R. E. Manning, J. McCauly, S. J. Monson, T. H. Oswald, and M. Pulupa, The electric antennas for the STEREO/WAVES experiment, Space Sci. Rev., doi:10.1007/s11214-007-9251-x, 2007.(1)

Bucci, F., A. Herique, W. Kofman, and D. Plettemeier, Polarimetric GPR: measurements of the backscattering by a discrete target in the Mie frequency region, Proceedings of the $5^{\text {th }}$ IWAGPR 2009, Grenade, Espagne, 2009.

Cecconi, B., X. Bonnin, S. Hoang, M. Maksimovic, S. D. Bale, J.-L. Bougeret, K. Goetz, A. Lecacheux, M. J. Reiner, H. O. Rucker, and P. Zarka, STEREO/Waves Goniopolarimetry, Space Sci. Rev., 172, doi: 10.1007/s11214-007-9255-6, 2007.(2)

Cecconi, B., X. Bonnin, S. Hoang, M. Maksimovic, S. D. Bale, J.-L. Bougeret, K. Goetz, A. Lecacheux, M.J. Reiner, H. O. Rucker, and P. Zarka, Stereo/Waves goniopolarimetry, Space Sci. Rev., 136, 549-563, doi:10.1007/s11214-007-9255-6, 2008. (3)

Fischer, G., W. Macher, H. O. Rucker, H. P. Ladreiter, D. F. Vogl, and the Cassini/RPWS Team, Wire-grid modeling of Cassini spacecraft for the determination of effective antenna length vectors of the RPWS antennas, in Planetary Radio Emissions $V$, edited by H. O. Rucker, M. L. Kaiser, and Y. Leblanc, Austrian Academy of Sciences Press, Vienna, 347-356, 2001.(4)

Karlsson, R., W. Macher, U. Taubenschuss, H. O. Rucker and the Cassini/RPWS team, In-flight calibration of the Cassini radio and plasma wave science (RPWS) antennas after the Huygens probe release, Proc. of HF07 - Nordic Shortwave Conference, 3.4.1.-3.4.9, Farö, Sweden, Aug 2007, 2007.5)

Krupar, V., M. Maksimovic, O. Santolik, and B. Cecconi, Direction finding on RPW (HFR): effects of the antenna tilt, Solar Orbiter Meeting, Toulouse, 2010.

Krupar, V., M. Maksimovic, O. Santolik, and B. Cecconi, An influence of antenna tilt angle to the RPW/Solar Orbiter direction finding, in Planetary Radio Emissions VII, 
edited by H.O. Rucker, W.S. Kurth, P. Louarn, and G. Fischer, Austrian Academy of Sciences Press, Vienna, this issue, 389-390, 2011.

Landmann, D., D. Plettemeier, and K.-H. Gonschorek, Messtechnische Validierung von Computersimulationen zur Lösung von inversen Strahlungskopplungsproblemen bei Antennen über geschichteten Untergrundstrukturen - Ein Beitrag zum Transmissionsexperiment CONSERT der Kometenmission Rosetta, EMV 2006 - Internationale Fachmesse und Kongress für Elektromagnetische Verträglichkeit, VDE-Verlag, 2006.

Leblanc, Y., and G. Daigne, The broadband Jovian kilometric radiation. Statistical properties and source model, in Planetary Radio Emissions, edited by H. O. Rucker and S. J. Bauer, Austrian Academy of Sciences Press, Vienna, 112-147, 1985.6)

Leblanc, Y., and G. Daigne, Jovian kilometric radiation: New results on polarization and beaming, J. Geophys. Res., 90, 12073, 1985.(7)

Lecacheux, A. and A. Ortega-Molina, Polarization and localization of the Uranian radio sources, J. Geophysic. Res., 92, 15148-15158, 1987. 8)

Macher, W., and T.H. Oswald, Radius correction formula for capacitances and effective length vectors of monopole and dipole antenna systems, Radio Sci., 46, RS1011, doi:10.1029/2010RS004446, 2011. (9)

Macher, W., B. Schraußer, G. Fischer, H. O. Rucker, H. Lammer, C. Kolb, and G. Kargl, Analysis of sounding antennas of the Mars Express MARSIS experiment, In: Proc. 2nd European Workshop on Exo/Astrobiology, edited by H. Sawaya-Lacoste, ESA Publ. Division, Noordwijk, ESA SP-518, 539-540, 2002.(10)

Macher, W., D. Plettemeier, H. O. Rucker, and G. Fischer, Wire-Grid simulations of the Mars Express/MARSIS antenna system, in: Planetary Radio Emissions VI, edited by H. O. Rucker, W. S. Kurth, and G. Mann, Austrian Academy of Sciences Press, Vienna, 483-490, 2006. (11)

Macher, W., T. Oswald, G. Fischer, and H. O. Rucker, Rheometry of multiport spaceborne antennas including mutual antenna capacitances and application to STEREO/WAVES, Meas. Sci. Techn., 18, 3731-3742, 2007.(12)

Ortega-Molina, A. and G. Daigne, Polarization response of two crossed monopoles on a spacecraft, Astron. Astrophys., 130, 301-310, 1984.

Ortega-Molina, A., and A. Lecacheux, Polarization response of the Voyager-PRA experiment at low frequencies, Astron. Astrophys., 229, 558-568, 1990.13)

Oswald, T.H., Antennas in plasma: numerical calculation, Ph.D. thesis, Karl-FranzensUniversity Graz, pp 164, 2010.

Oswald, T., W. Macher, G. Fischer, H. O. Rucker, J.-L. Bougeret, M. L. Kaiser, and K. Goetz, Numerical analysis of the STEREO WAVES antennas: First results, in Planetary Radio Emissions VI, edited by H. O. Rucker, W. S. Kurth, and G. Mann, Austrian Academy of Sciences Press, Wien, 475-482, 2006.(14) 
Oswald, T.H., H. O. Rucker, W. Macher and the Solar Orbiter RPW team, Aspects of the RPW antennas of solar orbiter, Proc. of the Second Solar Orbiter Workshop, ESA SP-641, 2007.15)

Oswald, T., W. Macher, H. O. Rucker, G. Fischer, U. Taubenschuss, J.-L. Bougeret, A. Lecacheux, M. L. Kaiser, and K. Goetz, Various Methods of calibration of the STEREO/WAVES antennas, Adv. Space Res., 43, 355-364, 2009.(16)

Panchenko, M., Polarimetry of auroral kilometric radiation with a triaxial non-orthogonal antenna system, Radio Sci., 39, RS 6010, doi:10.1029/2004RS003039, 2004.17)

Panchenko, M., H. O. Rucker, W. Macher, B. Cecconi, T. H. Oswald, and G. Fischer, STEREO/WAVES antennas calibrated by AKR, EPSC 2010-872, EPSC Rome, Sept 2010. (18)

Riddle, A. C., Antenna pattern testing on spacecraft model, Rep. MEM-MJS-76-S100, Lab. for Extraterr. Phys., NASA Goddard Space Flight Center, Greenbelt, MD., 1976.(19)

Rucker, H. O., W. Macher, R. Manning, and H. P. Ladreiter, Cassini model rheometry, Radio Sci., 31, 1299-1311, 1996.20)

Rucker, H. O., W. Macher, and S. Albrecht, Experimental and Theoretical Investigations on the Cassini RPWS Antennas, in Planetary Radio Emissions IV, edited by H. O. Rucker, S. J. Bauer, and A. Lecacheux, Austrian Academy of Sciences Press, Vienna, 327-337, 1997.21

Rucker, H. O., W. Macher, G. Fischer, T. Oswald, J.-L. Bougeret, M. L. Kaiser, and K. Goetz, Analysis of spacecraft antenna systems: Implications for STEREO/WAVES, Adv. Space Res., 36, 1530-1533, 2005.22

Rucker, H. O., T. H. Oswald, M. Sampl, and D. Plettemeier, Solar Orbiter RPW antennas numerical calibrations: An update, RPW $7^{\text {th }}$ team meeting Toulouse, 2010.(23)

Sampl, M., W. Macher, C. Gruber, T. Oswald, H. O. Rucker, and M. Mogilevsky, Resonance spacecraft antenna calibration: Rheometry and numerical simulations, in 2009 Loughborough Antennas and Propagation Conference, Burleigh Court Conference Centre, Loughborough University, United Kingdom, doi:10.1109/LAPC.2009. 5352452, 2009.(24)

Sampl, M., W. Macher, C. Gruber, T. Oswald, and H. O. Rucker, Rheometry, the effective length vector and the Resonance mission, E \& I, 5, 28-32, 2009.25)

Sampl, M., W. Macher, C. Gruber, T. Oswald, H. O. Rucker, M. Mogilevsky, Calibration of electric field sensors onboard the resonance Satellite, IEEE Transactions of Antennas and Propagation, in press, 2011.26)

Sayre, E., Res. Rep. Contract NAS5-24008, MOD-21, AVCO Corporation, 1976.27)

Vogl, D. F., H. P. Ladreiter, P. Zarka, H. O. Rucker, W. Macher, W. S. Kurth, D. A. Gurnett, and G. Fischer, First results on the calibration of the Cassini RPWS antenna 
system, in Planetary Radio Emissions V, edited by H. O. Rucker, M. L. Kaiser, and Y. Leblanc, Austrian Academy of Sciences Press, Vienna, 357-365, 2001.28

Vogl, D. F., B. Cecconi, W. Macher, P. Zarka, H. P. Ladreiter, P. Fedov, A. Lecacheux, T. Averkamp, G. Fischer, H. O. Rucker, D. A. Gurnett, W. S. Kurth, and G. B. Hospodarsky, In-flight calibration of the Cassini-RPWS antenna system for direction finding and polarization measurements, J. Geophys. Res., doi: 10.1029/2003JA010261, 109, 2004.29)

Wang, L., and T.D. Carr, Recalibration of the Voyager PRA antenna for polarization sense measurement, Astron. Astrophys., 281, 945-954, 1994.(30)

Zaslavsky, A., N. Meyer-Vernet, S. Hoang, M. Maksimovic, and S. D. Bale, On the antenna calibration of space radio instruments using the galactic background: General formulas and application to STEREO/WAVES, Radio Sci., 46, RS2008, doi: 10.1029/2010RS004464, 2011. (31) 
